\title{
Zero-crossing angle in the $n p$ analyzing power at medium energies and its relation to charge symmetry
}

\author{
T. S. Bhatia, G. Glass, J. C. Hiebert, L. C. Northcliffe, and W. B. Tippens \\ Texas A\&M University, College Station, Texas 77843
}

B. E. Bonner and J. E. Simmons

Los Alamos National Laboratory, Los Alamos, New Mexico 87545

\author{
C. L. Hollas, C. R. Newsom, ${ }^{*}$ P. J. Riley, and R. D. Ransome \\ University of Texas, Austin, Texas 78712
}

(Received 21 April 1981)

\begin{abstract}
The angle at which the analyzing power for free $n p$ scattering becomes zero, the zero-crossing angle $\theta_{0}$, has been measured simultaneously for the $\vec{n} p$ and $n \vec{p}$ scattering processes at 425,565 , and $665 \mathrm{MeV}$ incident neutron energies. A rather strong energy dependence of the zerocrossing angle is found. Knowledge of this energy dependence is important in designing an experiment which tests for charge symmetry breaking forces by comparing high precision measurements of $\theta_{0}(\vec{n} p)$ and $\theta_{0}(n \vec{p})$.
\end{abstract}

[NUCLEAR REACTIONS $p(n, p) n, E=425,565$, and $665 \mathrm{MeV}$; polarized neutron beam, polarized proton target; measured analyzing powers $A(E, \theta)$; deduced zero-crossing angles; charge symmetry.

Except for some indirect evidence, primarily from the comparison of binding energies of mirror nuclei, no clear-cut violation of charge symmetry of nuclear forces has yet been detected. ${ }^{1}$ As noted by Cheung, Henley, and Miller, ${ }^{2} n-p$ elastic scattering provides a convenient means to test for forces which cause isospin mixing in the two-body system, known in the literature as class IV charge symmetry breaking (CSB) forces. ${ }^{2}$ It was pointed out by Wolfenstein ${ }^{3}$ that for $n-p$ scattering, isospin conservation dictates the equivalence of the polarization $P_{n}(\theta)$ of neutrons scattered at any angle $\theta$ and the polarization $P_{p}(\pi-\theta)$ of the corresponding recoiling protons, i.e.,

$$
P_{n}(\theta)=P_{p}(\pi-\theta)
$$

As stated by Cheung, Henley, and Miller, ${ }^{2}$ class IV CSB forces break the above equality. The expected differences due mainly to electromagnetic effects and $\rho^{0}-\omega$ mixing are of the order of several tenths of one percent. Unfortunately the measurement of absolute polarizations of neutrons and protons with an accuracy of better than $1 \%$ is difficult.

In order to test for CSB forces, an alternate experimental approach is to attempt to verify the equality

$$
A(\vec{n} p)=A(n \vec{p})
$$

where $A(\vec{n} p)$ is the analyzing power with incident polarized neutron beam at given incident kinetic energy and neutron c.m. angle and $A(n \vec{p})$ is the analyzing power at the same energy and angle with polarized proton target. These analyzing power values are obtained from the experimentally measured asymmetries if the neutron beam polarization for $\vec{n} p$ scattering and the target proton polarization for $n \vec{p}$ scattering are known. Although the scattering asymmetries can be measured with high accuracy, the precision of the deduced analyzing powers will be limited by the accuracy with which the neutron beam and the target polarizations are known. Experimentally, it is difficult to calibrate the neutron beam and the proton target polarizations to better than a few percent. This implies difficulty in testing for the presence of CSB forces through comparisons of the magnitudes of analyzing powers.

It is possible that CSB forces may have an effect on the shape of the angular distribution of the analyzing powers that might be discerned experimentally. Gersten ${ }^{4}$ has discussed isospin nonconservation in $n p$ scattering and notes that small contributions to the singlet-triplet transitions, proportional to the relative proton-neutron mass difference, are obtained in the charge exchange one-pion exchange (OPE). In his Fig. 7 he indicates a slight shape difference between $P_{n 0}(\theta)$ and $P_{0 n}(\theta)$ [having the same meaning as the symbols in Eq. (1) above] at $325 \mathrm{MeV}$ which arises from Coulomb and pion and nuclear mass differ- 
ences. One manifestation of this shape difference is a shift of the zero-crossing angle, the angle at which the analyzing power $A$ becomes zero. The zerocrossing angle $\theta_{0}$ is independent of the neutron beam polarization in $\vec{n} p$ scattering and the target polarization in $n \vec{p}$ scattering. Such a zero-crossing angle can, in principle, be measured to high precision without absolute knowledge of the beam or target polarizations. The comparison of $\theta_{0}(\vec{n} p)$ and $\theta_{0}(n \vec{p})$ can therefore lead to a significant test for the CSB forces. ${ }^{5}$ From the data obtained in our recent experiment, ${ }^{6}$ in which polarized neutrons were scattered from a polarized proton target, the asymmetries for $\vec{n} p$ and $n \vec{p}$ scattering processes can be obtained by averaging over the target and beam polarizations, respectively, and from the measured angular distributions of the asymmetries the zero-crossing angles $\theta_{0}(\vec{n} p)$ and $\theta_{0}(n \vec{p})$ can be extracted. In this Communication the results of these zero-crossing angle measurements are reported. A rather strong energy dependence of the zero-crossing angle for analyzing power in free $n p$ scattering is found. This energy dependence is compared with the predictions from an energy dependent phase shift solution. ${ }^{7}$

The results reported here were obtained as a byproduct of a polarized beam-polarized target experiment, the purpose of which was to measure the spin correlation parameters $A_{\mathrm{NN}}$ in free $n p$ scattering. The polarized neutron beam was obtained by collimating the neutrons produced at $20^{\circ}$ lab in $800 \mathrm{MeV}$ unpolarized proton bombardment of a liquid deuterium $\left(\mathrm{LD}_{2}\right)$ target. This neutron beam has a broad spectrum of momenta. Its polarization, measured in a separate experiment, was found to be $\sim 0.20$ for almost the entire momentum range. ${ }^{8}$ Periodic reversal of the neutron beam polarization was achieved with the aid of precession magnets placed along the beam axis. This polarized neutron beam was scattered from a polarized proton target, ${ }^{9}$ the polarization of which was vertical, of magnitude $\sim 0.80$, and was periodically reversed. Simultaneous measurements of the scattering angle and momentum of the recoiling proton and the direction and velocity of the scattered neutron enabled unambiguous selection of the elastic $n p$ events. Such kinematic overdetermination of the two-body final state drastically reduces the backgrounds due to scattering from the nonhydrogen components of the target material and the walls of the polarized target cryostat. The details of the experimental arrangement will be described elsewhere. Figure 1 shows a typical incident neutron energy spectrum, obtained from the measured momenta of the recoiling protons for the elastic $n p$ events. The $n p$ elastic scattering data were analyzed in three bins, corresponding to mean incident neutron kinetic energies of 425,565 , and $665 \mathrm{MeV}$, respectively. The bin widths were $100 \mathrm{MeV}$ except for the highest energy bin where it was somewhat less. For each energy

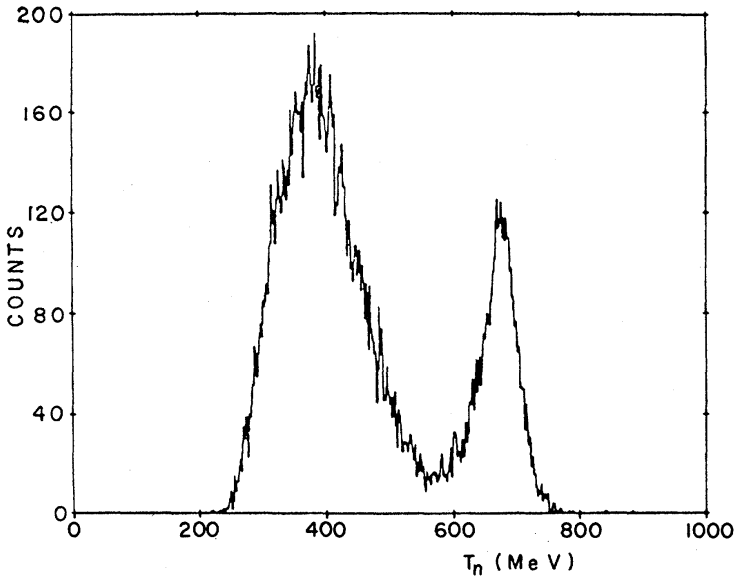

FIG. 1. The neutron energy spectrum obtained from the measurement of the momenta of the recoiling protons for free $n p$ elastic scattering at $110^{\circ} \mathrm{c} . \mathrm{m}$. The cutoff just above $200 \mathrm{MeV}$ is of instrumental origin.

bin, the angular distribution of the asymmetry, $\epsilon(\theta)=P_{T} A(\theta)$, for the $n \vec{p}$ scattering process was extracted by averaging the results obtained with the two states of the neutron beam polarization, so as to simulate an unpolarized beam. Alternatively, for each energy bin, the angular distribution of the asymmetry for the $\vec{n} p$ scattering process $\epsilon(\theta)=P_{B} A(\theta)$ was extracted by averaging over the results obtained with the two orientations of proton target polarization. $P_{T}$ and $P_{B}$ are target and beam polarizations, respectively.

Polynomial fits to these angular distrubutions were used to determine the zero-crossing angles for the two processes at each of the three energies. The zero-crossing angles thus obtained for $\vec{n} p$ and $n \overrightarrow{\vec{p}}$ scattering at 425,565 , and $665 \mathrm{MeV}$ are shown in Fig. 2. Also shown in Fig. 2 is the predicted energy dependence of the zero-crossing angle for free $n p$ analyzing power based on the energy dependent phase shift solution, WI80, of Arndt and VerWest. ${ }^{7}$ It is seen that the measured $\theta_{0}(n \vec{p})$ values agree well with those for $\theta_{0}(\vec{n} p)$ for each of the three energy bins reported and that the measured values are generally in agreement with the phase shift prediction. While Gersten ${ }^{4}$ predicts a zero-crossing angle difference of over $2^{\circ}$ for $325 \mathrm{MeV}$, the magnitude of the effect predicted by Cheung et al. ${ }^{2}$ for $300 \mathrm{MeV} n p$ scattering is much smaller. The accuracy of these measurements is almost sufficient to test for a zerocrossing difference of the magnitude predicted by Gersten ${ }^{4}$ but would need to be improved considerably to measure effects of the magnitude predicted by Cheung et al. ${ }^{2}$

It should be noted that the precision with which the zero-crossing angle can be extracted from a given 


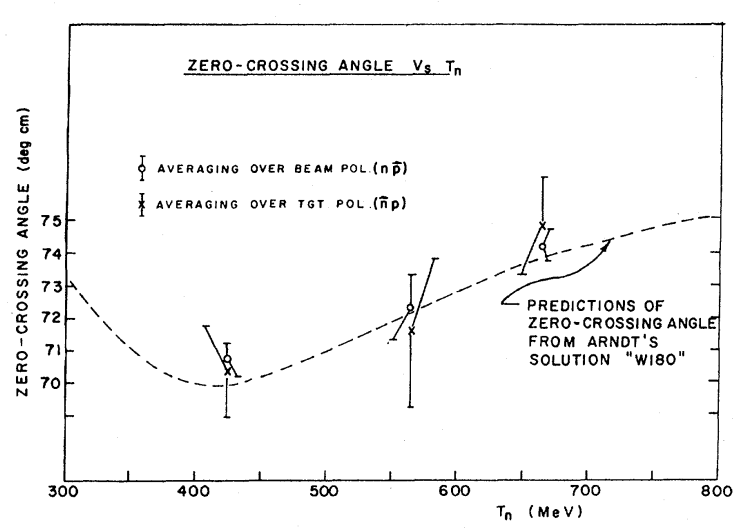

FIG. 2. The zero-crossing angles $\theta_{0}(n \vec{p})$ and $\theta_{0}(\vec{n} p)$ for 425,565 , and $665 \mathrm{MeV}$ energy bins. The curve shows the predicted energy dependence for $300-800 \mathrm{MeV}$ based on the energy dependent phase shift solution WI80 of Arndt and VerWest.

angular distribution of the measured asymmetry, $\epsilon(\theta)$ depends not only on the errors $\Delta \epsilon(\theta)$ associated with the measurements of asymmetry, but also on the slope of $\epsilon(\theta)$ near the zero-crossing angle. This slope increases with the use of higher proton target polarization for $n \vec{p}$ scattering and higher neutron beam polarization for $\vec{n} p$ scattering. Thus even though the asymmetries for the two processes were measured with the same precision in this experiment, the accuracy of zero-crossing angle extracted for $\vec{n} p$ scattering is not as good as that for $n \vec{p}$ scattering because the neutron beam polarization was only $\sim 0.20$ whereas the target polarization was $\sim 0.80$. The accuracy of the $\theta_{0}(\vec{n} p)$ measurement could be improved considerably by use of a neutron beam of higher polarization. One way of obtaining such a beam is indicated by a recent measurement ${ }^{10}$ of the polarization transfer parameter $K_{L L}$ in the reaction $\vec{p} d \rightarrow \vec{n} x$ at $0^{\circ}$ and $800 \mathrm{MeV}$. The large transfer parameter observed, $K_{L L}=0.64 \pm 0.03$, would provide a useful beam of polarized neutrons $\left(P_{n} \sim 0.5\right)$ at LAMPF if the incident polarized proton beam intensity could be increased by an order of magnitude.

Knowledge of the energy dependence of the zerocrossing angle, which is seen in Fig. 2, is important for the purpose of planning an experiment for testing for CSB forces by high precision measurement of $\theta_{0}(\vec{n} p)$ and $\theta_{0}(n \vec{p})$. From 400 to $665 \mathrm{MeV}, \theta_{0}$ changes by $\sim 3.5^{\circ}$. The uncertainties in the knowledge of the incident neutron beam energies could lead to significant errors if $\vec{n} p$ and $n \vec{p}$ scattering were studied in separate experiments, except near $425 \mathrm{MeV}$ where the slope of $\theta_{0}$ vs $T_{n}$ is zero. Such problems do not arise if, as in the present work, the data for the two processes are obtained simultaneously in an $\vec{n} \vec{p}$ scattering experiment, since the beam energies for the deduced $\vec{n} p$ and $n \vec{p}$ scattering would then be identical.

We would like to thank the Cryogenics group at LAMPF for their expert assistance in maintaining the liquid deuterium neutron production target during the course of this experiment. Jan Boissevain's help in all phases of the polarized target operation is gratefully acknowledged. One of us (T.S.B.) wishes to thank Leon Heller and Alex Gersten for helpful discussions. This work was supported by the U.S. Department of Energy.
"Present address: Laboratoire National Saturne, 91191 Gifsur-Yvette Cèdex, France.

${ }^{1}$ E. M. Henley, in Isospin in Nuclear Physics, edited by D. H. Wilkinson (North-Holland, Amsterdam, 1969), p. 15.

${ }^{2}$ C. Y. Cheung, E. M. Henley, and G. A. Miller, Nucl. Phys. A 305, 342 (1978); A348, 365 (1980).

${ }^{3}$ L. Wolfenstein, Ann. Rev. Nucl. Sci. 6, 43 (1956).

${ }^{4}$ A. Gersten, Phys. Rev. C $\underline{18}, 2252$ (1978).

${ }^{5}$ S. E. Vigdor et al., in Proceedings of the Fifth International Symposium on Polarization Phenomenon in Nuclear Physics, Santa Fe, 1980, edited by G. G. Ohlsen (AIP, New York,
1981), p. 1455.

${ }^{6}$ T. S. Bhatia et al., in Ref. 5, p. 123.

${ }^{7}$ R. A. Arndt and B. VerWest (private communications).

${ }^{8}$ T. S. Bhatia et al., in Ref. 5, p. 871.

${ }^{9}$ The polarized proton target used consists of a $40 \mathrm{~cm}^{3}$ cryostat filled with $\mathrm{Cr}$ doped propanediol, cooled to $\sim 0.5$ $\mathrm{K}$ placed between the poles of a " $C$ " magnet $(25 \mathrm{kG})$. The protons in the target material are dynamically polarized to about $80 \%$ using optical pumping.

${ }^{10}$ P. J. Riley et al., in Ref. 5, p. 874; Phys. Lett. (in press). 